\title{
Trends and patterns in urgent pediatric otolaryngology inter-hospital referrals in Saudi Arabia
}

\author{
Marwa A. Algadi, MBBS, Alanoud A. Alshathri, MBBS, Rawan S. Alsugair, MBBS, Mohrah A. Alyabis, MBBS,
} Saad A. Alsaleh, MBBS, FRCSC, Nawfal A. Aljerian, MBBS, MHPE.

\begin{abstract}

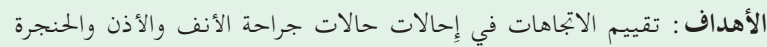

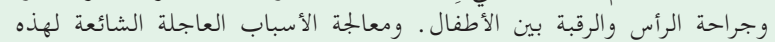

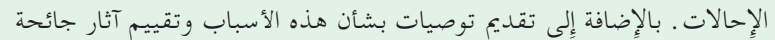

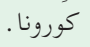

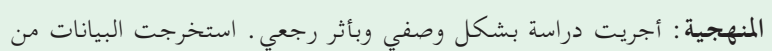

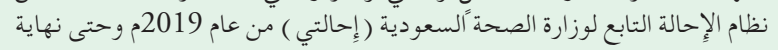

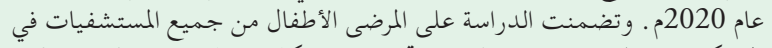

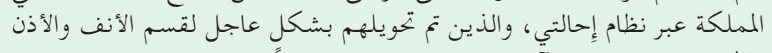

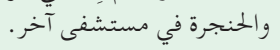

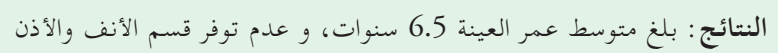

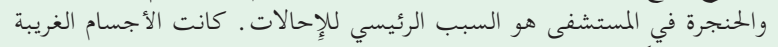

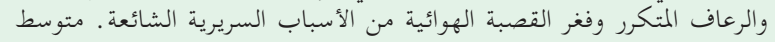

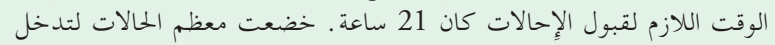

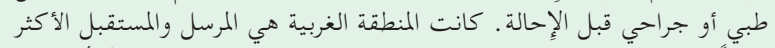

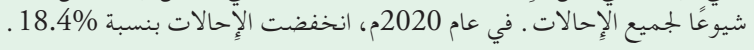
الحلاصة : تمت التوصية بالتوسع في خدمات جراحة الأنف والأذن والحنجرة في المناطق ذات الإحالات المرتفعة.
\end{abstract}

Objectives: To assess trends in otolaryngology-head and neck surgery (ORL-HNS) referrals among pediatric patients, and to address common urgent causes of ORLHNS referrals in this population. Additionally, to give recommendations regarding these causes, and assessing the impacts of coronavirus desease-19 on such referrals.

Methods: A retrospective descriptive study were the data was extracted from the referral system of the Saudi Ministry of Health (Ehalati), from 2019-2020. Pediatric patients from all hospitals across Saudi Arabia with problems related to ORL-HNS who have been referred urgently to other hospitals were included.

Results: A total of 1318 urgent ORL-HNS referrals were collected. The average age of the sample was 6.5 years, with the unavailability of specialty being the major cause for referrals. Foreign bodies, recurrent epistaxis, and tracheostomy were also common clinical causes. The average time for accepting referrals was 21 hours.
Most cases had medical or surgical intervention prior to referral. The Western region of Saudi Arabia was the most common sender and receiver of all referrals. In 2020 , referrals decreased by $18.4 \%$.

Conclusion: Maternity and pediatric hospitals make up the largest number of referring hospitals for ORL-HNS urgent cases. Expanding ORL-HNS services has been recommended in highly demanding areas. standards for urgent ORL-HNS referrals may limit inappropriate urgent referrals.

Keywords: trend, ORL-HNS, pediatric, referrals, urgent

Saudi Med J 2022; Vol. 43 (1): $91-97$

doi: 10.15537/smj.2022.43.1.20210710

From the College of Medicine (Algadi, Alshathri, Alsugair, Alyabis), Almaarefa University, from the Department of Otolaryngology Head and Neck Surgery (Alsaleh), College of Medicine, King Saud University, and from Medical Referrals Center (Aljerian), Ministry of Health, King Saud bin Abdulaziz University for Health Sciences, Riyadh, Kingdom of Saudi Arabia.

Received 4th September 2021. Accepted 23rd November 2021.

Address correspondence and reprint request to: Dr. Marwa A. Algadi, Medical Intern, College of Medicine, Almaarefa University, Riyadh, Kingdom of Saudi Arabia. E-mail: algadi.marwa1@gmail.com ORCID ID: https://orcid.org/0000-0002-8910-6543

Complaints related to the ear, nose, and throat $($ ENT) are among the most frequant in the pediatric emergency department (ED). ${ }^{1}$ Often, pediatricians and otolaryngology-head and neck surgery (ORL-HNS) specialists are called to assess otorhinolaryngology disorders that require intervention. These cases can result from infection, trauma, and foreign bodies (FB) or late diagnosis of congenital problems. ${ }^{2}$ Some 
cases may require additional management that some hospitals lack the resources to provide due to a lack of appropriate instruments, expert specialists, or patients' families requesting another specialist or service. These reasons push hospitals to refer these patients to other hospitals, which can be costly, time-consuming, and disabling for parents and the patient, especially when referring to a hospital located outside of their region.

Approximately $61 \%$ of ORL-HNS cases are emergencies, and only $9 \%$ of them require medium to high interventions. ${ }^{3}$ Swallowing of FB is among the most common pediatric ORL-HNS emergencies. The majority of nose FB can be successfully removed in the ED. However, there is a high failure rate in the removal of $\mathrm{FB}$ from the ear which should be referred directly to ORL-HNS services. ${ }^{4}$ Timing of intervention is important for reducing mortality and morbidity in such cases. ${ }^{5}$

A study carried out in Port Harcourt revealed that ORL-HNS emergencies are common, especially in the pediatric age group. ${ }^{6}$ Foreign bodies and trauma were among the most common causes. Pediatric ORL-HNS emergencies are common in Nigeria too, yet many involve different diseases that require surgical intervention. ${ }^{7}$ The causes are different for adults. Peripheral vertigo and otitis externa are the most common causes for referrals from the ED to ORL-HNS clinics. ${ }^{8}$ In the rural area of Gujarat, facio-maxillary trauma forms the majority of ORL-HNS emergencies. ${ }^{9}$ Otolaryngologists covering EDs should be familiar with this population in terms of practice and referrals. ${ }^{8}$ Establishing clear criteria for urgent ORL-HNS referrals helps other care providers refer more appropriately. ${ }^{10}$ A nationally representative general practice database in the Netherlands showed a stable trend in incidence, rates, and referrals of common ORL-HNS problems among pediatric. ${ }^{2}$

In Saudi Arabia, there are limited researches regarding medical referrals. ${ }^{11}$ Multiple studies had evaluated the quality and pattern of referrals from primary care health units to the hospitals, in Riyadh, $\mathrm{Al}$ Qassim, and the Southeastern region. ${ }^{12-14}$ Another study discussed a specific pattern regarding diabetic patients' referrals. ${ }^{15}$ There is a lack of researchers that discuss referrals in the otolaryngology field, inter-hospital referrals, and among the pediatric population as well. For this reason, this study aimed to assess these trends and address the common causes of pediatric ORL-HNS

Disclosure. Authors have no conflict of interests, and the work was not supported or funded by any drug company. referrals. Furthurmore, we provide recommendations overall and assess the impact of coronavirus desease-19 (COVID-19) on such referrals.

Methods. A retrospective and descriptive study involving pediatric patients with problems related to ORL-HNS who have been referred urgently to other hospitals. A total of 1318 referrals were extracted retrospectively, from January 2019 until December 2020. The data was retrieved from the referral program (Ehalati), an e-system under the Ministry of Health $(\mathrm{MOH})$ of Saudi Arabia, which manages the process of referring patients between all hospitals inside the Kingdom, public and private of the same region or other regions. The main data that the system provides is demographics, diagnosis, cause of referral, time spent in accepting the referral request, areas and hospitals of referral's senders and receivers, and any medical or surgical intervention conducted in the ED before the referral.

The study was conducted across all hospitals in Saudi Arabia, registered in the system. All accepted urgent cases of patients under 18 and referred for another ORL-HNS services in other hospitals were included in this study. Life-threatening and out-patient cases, patients older than 18 years old, incomplete referrals, and referrals unrelated to ORL-HNS were excluded. Ethical approval was obtained from the Ethical Committee of Almaarefa University, Riyadh, Saudi Arabia.

Statistical analysis. Figures and graphs were generated by using Microsoft Excel. All statistical analysis tests were carried out by using Statistical Package for the Social Sciences for Windows, version 26 (IBM Corp., Armonk, NY, USA).

Results. A total of 1318 referrals were included, with a mean age of 6.5 years. Male patients represented $55.4 \%$ of the referrals. There were 726 ORL-HNS referrals in 2019 (55\% of all urgent pediatric referrals), while in 2020 referrals had decreased to 592 (45\%). The average time of referral acceptance was 21 hours. The majority of cases $(60 \%)$ had medical or surgical interventions prior to referral. The major cause of referrals was unavailability of speciality (Department of ORL-HNS) within the sender hospital (57.4\%), followed by unavailability of specialist doctors (35.7\%), and further followed by lack of required devices (5.7\%). Only $1 \%$ were due to a lack of beds (Figure 1). Most cases $(95.3 \%)$ were sent for general ORL-HNS services, few cases $(2.5 \%)$ were sent for audiology, and even fewer for phonetics $(1.1 \%)$, and head and neck surgery $(0.9 \%)$. 
Foreign bodies were the most common clinical cause for referrals, seen in $343(26.1 \%)$ cases, followed by epistaxis (8.7\%), and tracheostomy (8.4\%; Figure 2). Among FB cases, 133 (38.7\%) were ingested, 74 (21.5\%) were nasal, $62(18 \%)$ were in the ear, $17(4.9 \%)$ were aerodigestive, and $5(1.4 \%)$ cases were suspected FB. Regarding FB materials, $48.9 \%$ of them were unknown or not mentioned by the sender hospitals, $19.2 \%$ were coins, and $12.8 \%$ were food particles (Figure 3 ).
Trauma and fractures were common causes of referral. There were a total of 70 trauma cases, of which 45 were nasal, 12 were related to the ear, and 9 to the face. There were 66 fracture cases, the majority (92\%) being nasal fractures. The most common upper airway manifestations were 30 cases of stridor and 24 cases of non-mechanical upper airway obstruction.

Regarding senders and receivers, areas are categorized according to regions: West (38.3\%), South $(20.3 \%)$,

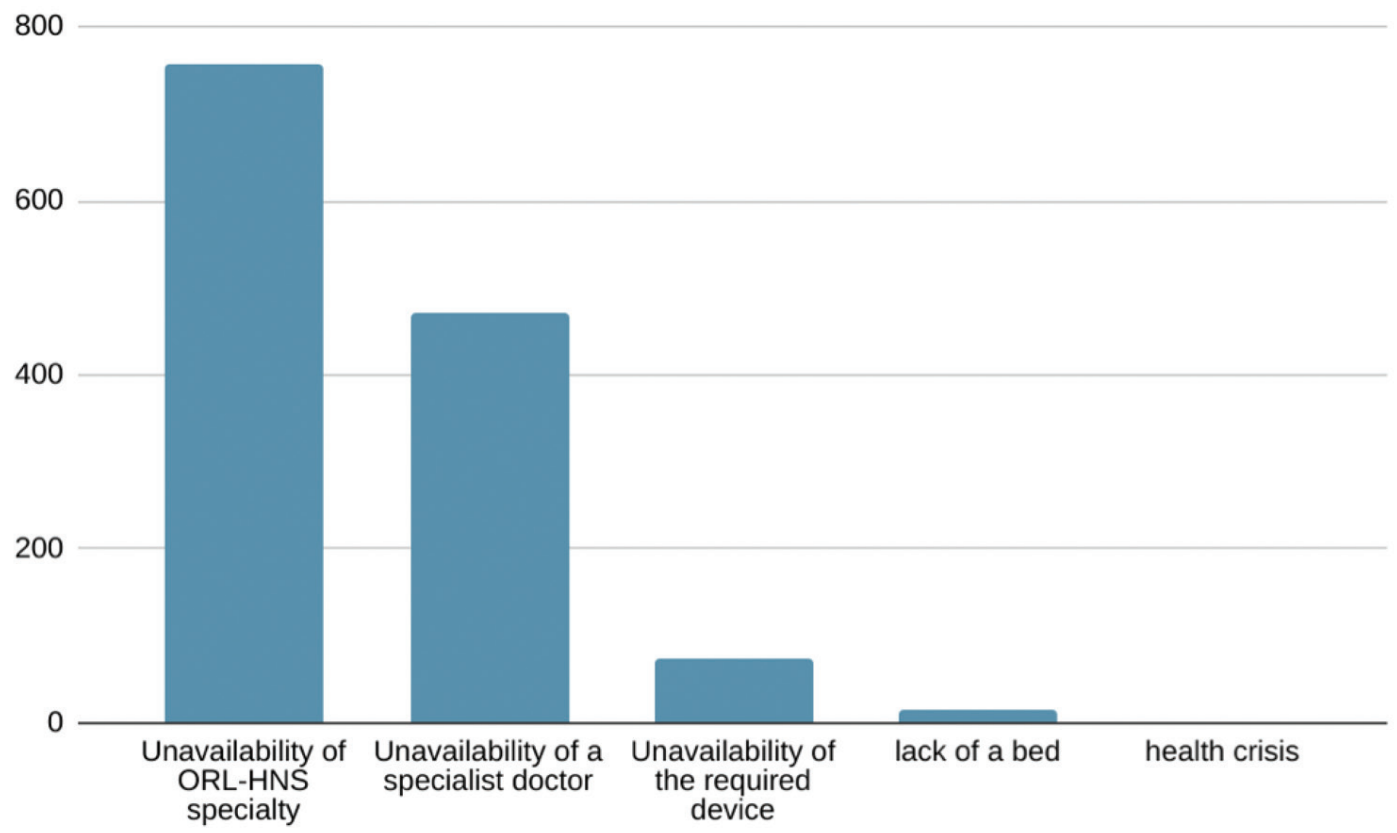

Figure 1 - Causes of urgent inter-hospitals otolaryngology referrals among pediatric. ORL-HNS: otolaryngology-head and neck surgery

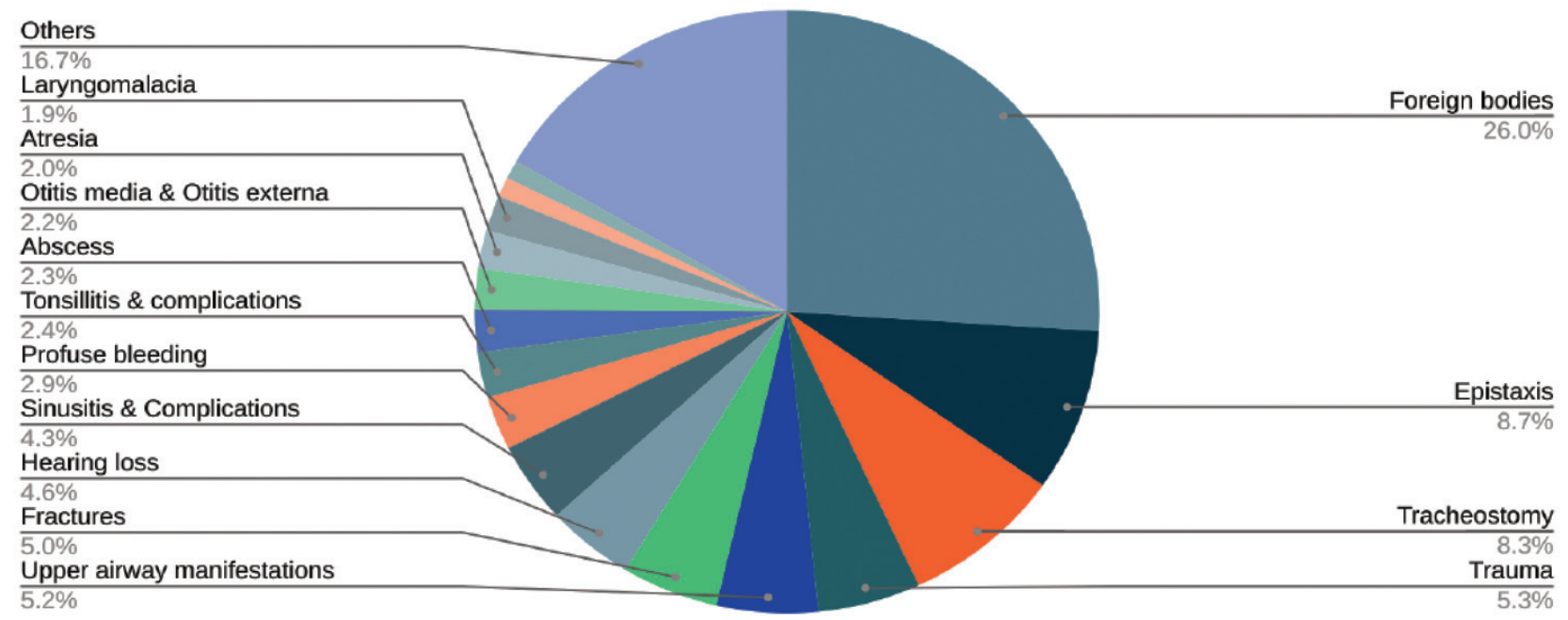

Figure 2 - Clinical causes of urgent inter-hospital otolaryngology referrals among pediatric. 
North (19.3\%), Central (16\%), and Eastern (6.2\%). The most common cities were Al Madinah Al Munawarah, Jazan, Al Qassim, North Borders, and Hafr Albatin. Referrals were usually received by hospitals within the same area, and a small number was referred outside the area (Figure 4).

Discussion. Pediatric ORL-HNS emergencies are one of the most common presentations in the ED. ${ }^{16}$ In many EDs, referring is the best choice that a physician can provide for the patient in order to ensure high-quality service and fewer complications in the future. On the other hand, referrals can be costly and time-consuming. Knowing the distribution of referrals according to regions contributes to providing better and faster services, and thus improving the quality of life for patients. Among the Western region, Al Madinah Al Munawarah, had the highest referral number during the past 2 years, approximately $70 \%$ of the Western region and $27 \%$ of total referrals across Saudi Arabia.

Although FB is the most clinical cause for referrals across Saudi Arabia, tracheostomy is the most common clinical cause in Al Madinah Al Munawarah (66 referrals, $18.7 \%$ ), representing $60 \%$ of total tracheostomy referrals in Saudi Arabia. Approximately 51.5\% were referred for tracheostomy, while $30.3 \%$ were referred for the assessment of tracheostomy tubes and 18.1\% for tracheostomy complications. "Tracheostomy" was not mentioned in previous studies as an observed reason for referring patients. ${ }^{10}$ However, tracheostomy requires

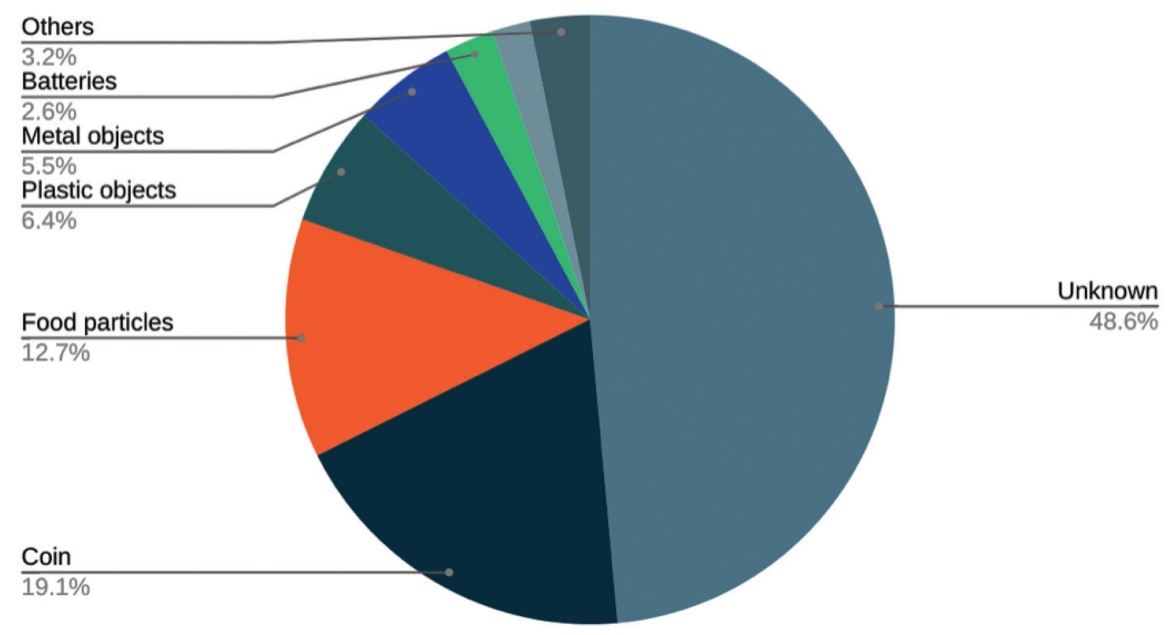

Figure 3 - Types of foreign bodies for which pediatric are referred to otolaryngology service in other hospitals.

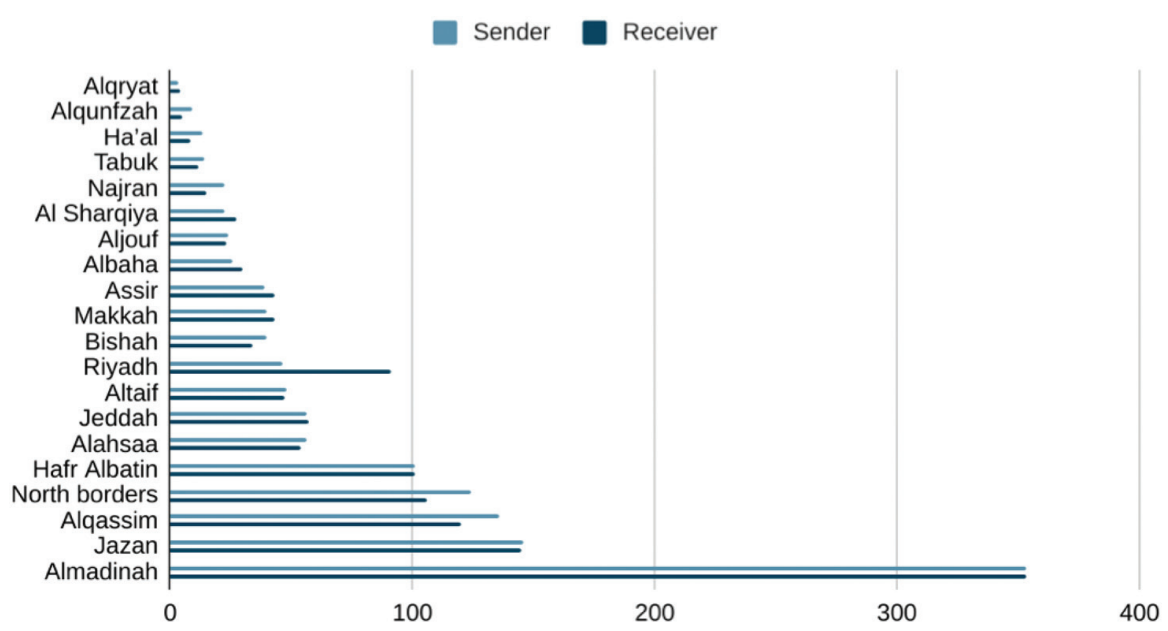

Figure 4 - The number of urgent pediatric otolaryngology inter-hospitals referrals, according to the regions of Saudi Arabia. 
special training in pediatric ENT. The increase in tracheostomy referrals in $\mathrm{Al}$ Madinah $\mathrm{Al}$ Munawarah, is mostly due to the low number of pediatric ENT specialists in the area. However, the official statistics regarding the specialized physicians in the area are limited.

Most referrals (61\%) were sent from maternity and pediatric hospitals, which helps explain the unavailability of specialty (49.2\%) and specialties (49\%) being the highest cause of referrals in Al Madinah Al Munawarah. Despite the high number of referrals sent from maternity and pediatric hospitals, only 2 cases were referred outside the area in the previous 2 years, with the average time to accept a referral within the area being 4 hours. This indicates that urgent ORL-HNS cases can still be managed within the area, regardless of their number.

A total of $204(15 \%)$ referrals took 24 hours to get accepted by other hospitals, yet the average wait time was 86 hours. Delayed acceptance has been found in Jazan (23\%), Riyadh (13\%), and Al Qassim (11\%). The majority of delayed referrals are due to the unavailability of specialties (68\%). Most of the delayed referrals (62\%) have not received any medical intervention by the sender hospitals. The mean age of patients with delayed referrals was 7 years old. Approximately $22 \%$ of these cases are due to nasal trauma and fractures, $10.2 \%$ for decreased hearing and hearing assessment, followed by epistaxis (7.3\%), and tracheostomy (7.3\%). This delay in acceptance can be explained by the relatively lower urgency of these cases, resulting in a wait time of one week to several weeks. Sender hospitals, however, tend to refer urgently cases of hearing assessment and aid devices to prevent any delay or restriction of language development in these cases. This is similar to a study carried out at St George's Hospital, London. ${ }^{10}$ The study revealed that otitis externa, recurrent epistaxis, and fractured noses are the most common causes of referral from general practitioners to the emergency ENT clinic. The study described those referrals as "inappropriate urgent referrals". ${ }^{10}$

Recurrent and frequent referrals of the same patients have been reported for $17 \%$ of total referrals, with a mean age of cases being 4 years, mostly coming from Al Madinah Al Munawarah (60\%). Tracheostomy and tracheostomy complications (20\%) are the most common clinical cause for frequent referrals too, followed by epistaxis (18\%). This recurrence in referrals is mostly due to the protocols in multiple hospitals, which refuse to continue the management of the case, deal with the complications, or simply due to persistent unavailability of the requested service.

In 2020, urgent ORL-HNS referrals decreased by $18.4 \%$ in comparison to 2019 , mostly due to COVID-19 and the changes in priority of medical services due to the pandemic. This resulted in restricting the medical services to only urgent cases. This was noticed especially in the first 4 months of the pandemic (from March until June; Figure 5). In the subsequent months, a brief increase in the numbers of referrals was seen, which meets the summer vacation in normal

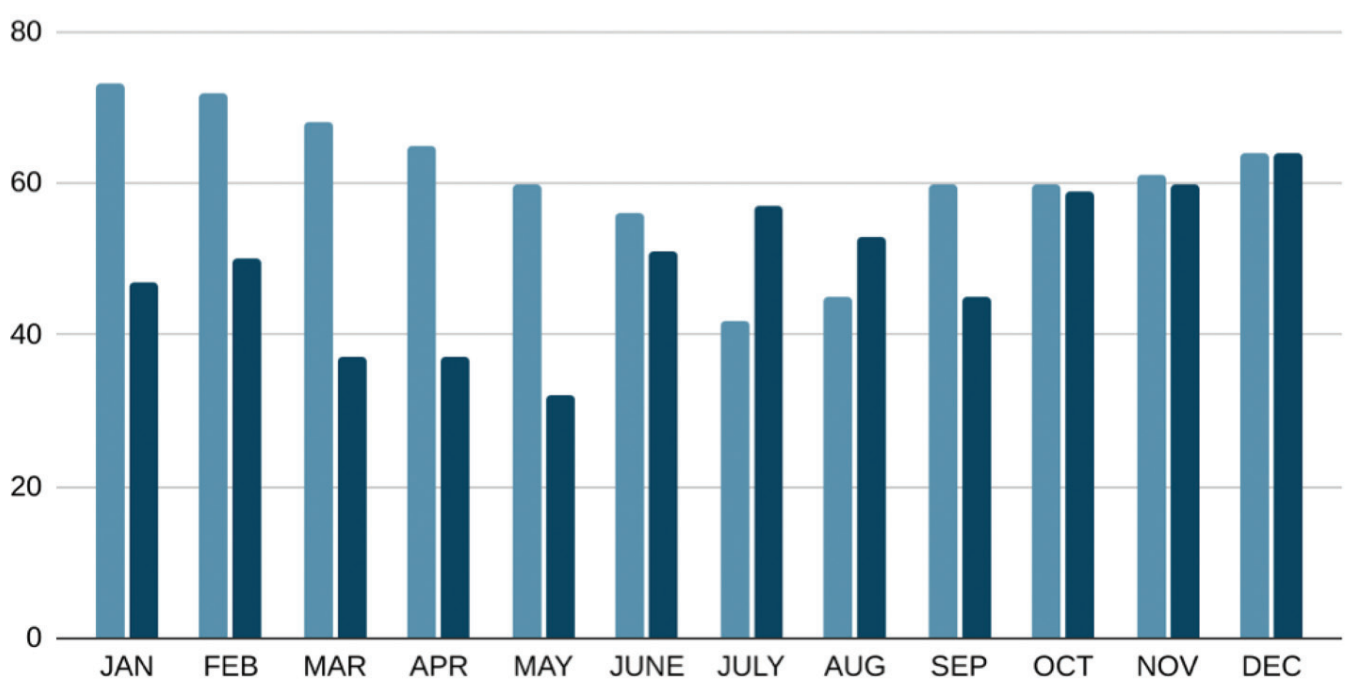

Figure 5 - The number of urgent pediatric inter-hospitals otolaryngology referrals, according to the months of 2019 and of 2020. 
circumstances. However, in 2020, this increase was mostly due to the lockdown and families spending their summer vacations inside Saudi Arabia. The average time to accept referrals had also decreased by 3 hours, reaching 20 hours on average compared to 23 hours in 2019. This decrease can be explained by the decrease in workload on ORL-HNS services during the pandemic and its restrictions, and therefore, faster responses to urgent referrals.

There was no change in the clinical causes during the pandemic; FB, epistaxis, and tracheostomy are the most common causes. Al Madinah Al Munawarah, also remained as the most frequent sender area. In comparison to 2019, FB referrals had increased by $6.5 \%$, epistaxis had decreased by $20.7 \%$, and fractures and trauma had decreased by $34.4 \%$. Referrals for tracheostomy showed an insignificant decrease by $2.4 \%$. The expected increase in FB during the lockdown, and increased time spent at home is known as household hazards. ${ }^{17}$ The decrease in other causes was related to curfew and decreased outdoor activities, and therefore decreased exposure to triggering factors. These findings were similar to the University of Eastern Piedmont ENT clinic, who found that during COVID-19 (from March until May), the number of referrals for FB increased by $25 \%$, decreased in epistaxis by $80 \%$ and nasal bone fracture by $70 \% .{ }^{18}$ Another study also showed a general decrease in ORL-HNS referrals from EDs by $93 \%$, and a decrease in epistaxis referrals by $83 \%{ }^{19}$

Study limitations. Our study encountered limited information regarding "time of arrival" for many of the cases, and limited ORL-HNS subspecialties included in the referral system (Ehalati). Subsequently, we recommend that the the Referral Center updates the arrival time on the system to be mandatory for all hospitals, and that they add more subspecialties to the system.

In conclusion, the unavailability of the specialty within the hospital is considered the main cause of urgent otolaryngology referrals in pediatric across Saudi Arabia. foreign bodies, epistaxis, and tracheostomy are the most common clinical reasons for referrals. In general, Western region hospitals are among the most sender and receiver hospitals. In 2020, and during the COVID-19 pandemic, referrals decreased in numbers without change in the causes.

The services of ORL-HNS should be expanded, especially in areas of high demand. In order to provide rapid access for urgent ORL-HNS cases and improve the quality of life for the patients, a pediatric ENT specialist in maternity and pediatric hospitals can be a reasonable solution since a large number of cases are referred from these hospitals. Clear criteria for urgent ORL-HNS cases are also recommended to reduce inappropriate referrals, and to save resources and time for true urgent cases.

Acknowledgment. The authors gratefully acknowledge Mr. Nayyaf Alharbi for his generous help in the statistical analysis. We also would like to thank Etqan (https://ksa-itqan.com/) and wordy (https://www.wordy.com/) for English language editing.

\section{References}

1. Al-Mazrou KA, Makki FM, Allam OS, Al-Fayez AI. Surgical emergencies in pediatric otolaryngology. Saudi Med J 2009; 30: 932-936.

2. Uijen JH, Bindels PJ, Schellevis FG, van der Wouden JC. ENT problems in Dutch children: trends in incidence rates, antibiotic prescribing and referrals 2002-2008. Scand J Prim Health Care 2011; 29: 75-79.

3. Furtado PL, Nakanishi M, Rezende GL, Granjeiro RC, de Oliveira TS. Clinic-epidemiological analysis of an otorhinolaryngology emergency unit care in a tertiary hospital. Braz J Otorhinolaryngol 2011; 77: 426-431.

4. Mackle T, Conlon B. Foreign bodies of the nose and ears in children. Should these be managed in the accident and emergency setting? Int J Pediatr Otorhinolaryngol 2006; 70: 425-428.

5. Rana AK, Kumar S, Sharma R, Sharma VK. Presentations of pediatric emergencies in a tertiary teaching hospital in North India: an overview. Indian J Otolaryngol Head Neck Surg 2019.

6. Ibekwe UM. Otorhinolaryngological emergencies in a tertiary hospital in Port Harcourt. Niger J Clin Pract 2017; 20: 606-609.

7. Adoga AA, Okwori ET, Yaro JP, Iduh AA. Pediatric otorhinolaryngology emergencies at the Jos University Teaching Hospital: study of frequency, management, and outcomes. Ann Afr Med 2017; 16: 81-84.

8. Garneau JC, Wasserman I, Konuthula N, Malkin BD. Referral patterns from emergency department to otolaryngology clinic. Laryngoscope 2018; 128: 1062-1067.

9. Yojana S, Mehta K, Girish M. Epidemiological profile of otorhinolaryngological emergencies at a medical college, in rural area of gujarat. Indian J Otolaryngol Head Neck Surg 2012; 64: 218-224.

10. Mahalingam S, Pepper C, Oakeshott P. Reducing inappropriate ENT referrals. Br J Gen Pract 2013; 63: 404-405.

11. Jarallah JS. Referral from primary care to hospitals in Saudi Arabia: 1) quality of referral letters and feedback reports. $J$ Family Community Med 1998; 5: 15-22.

12. Jarallah JS. The quality of referral letters in two health centers in Riyadh. Ann Saudi Med 1991; 11: 658-662.

13. Al-Alfi MA, Al-Saigul AM, Abed-Elbast AM, Sourour AM, Ramzy HA. Quality of primary care referral letters and feedback reports in Buraidah, Qassim region, Saudi Arabia. J Family Community Med 2007; 14: 113-117. 
14. Abdelwahid HA, Al-Shahrani SI, Elsaba MS, Elmorshedi WS. Patterns of referral in the family medicine department in Southeastern Saudi Arabia. Saudi Med J 2010; 31: 925-930.

15. Senitan M, Alhaiti AH, Gillespie J, Alotaibi BF, Lenon GB. The referral system between primary and secondary health care in Saudi Arabia for patients with type 2 diabetes: a systematic review. J Diabetes Res 2017; 2017: 4183604.

16. Stoner MJ, Dulaurier M. Pediatric ENT emergencies. Emerg Med Clin North Am 2013; 31: 795-808.
17. Oyama LC. Foreign bodies of the ear, nose and throat. Emerg Med Clin North Am 2019; 37: 121-130.

18. Campagnoli M, Cerasuolo M, Renna M, Dell'Era V, Valletti PA, Garzaro M. ENT referral from emergency department during COVID-19: a single-center experience. Ear Nose Throat J 2021: 145561320984560.

19. Osborne MS, Bentley E, Farrow A, Chan J, Murphy J. Impact of coronavirus disease 2019 on urgent referrals to secondary care otolaryngology: a prospective case series. J Laryngol Otol 2020: 1-4. 